\section{The genetic classification of prostate cancer: what's on the horizon?}
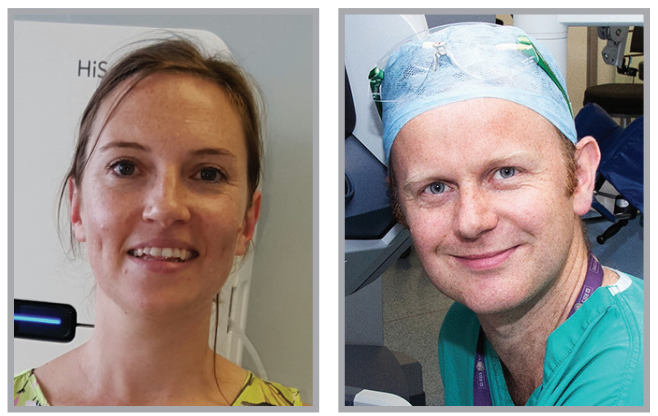

\author{
"It is now time to deliver on \\ the promises of stratified \\ medicine...”
}

First draft submitted: 4 December 2015; Accepted for publication: 8 December 2015; Published online: 9 February 2016

There are still fundamental aspects of prostate cancer diagnosis and treatment where advice is conflicting and gold standard care is unclear. Consider the use of prostatespecific antigen (PSA) diagnostic screening: conflicting evidence from large, carefully designed studies in Europe and the USA has led to confusion over whether nationwide screening programs are advisable for men $>55$ years $[1,2]$. Or in the UK, where the ProtecT trial seeks to determine the best course of treatment for localized prostate cancer - radiotherapy, surgery or 'active surveillance', and will report in 2016 [3]. On both sides of the Atlantic, there is still heavy reliance on histopathological scoring of tumors by Gleason grade. While Gleason scores of 6 or lower are now generally considered indolent, Gleason scores $\geq 7$ (conventionally grouped into 'intermediate' Gleason 7 or 'high risk' Gleason 8-10) alongside PSA levels and $T$ stage [4] present a problem, as many men with these tumors have outcomes that confound their risk prediction. A recent population-based trial from Sweden [5] has attempted to enhance PSA-based screening with a stepwise algorithm incorporating additional tests including an single nucleotide polymorphism array as a genetic component and such approaches are likely to form the basis of 'next-generation' screening [6]. In this context, molecular profiling studies of prostate cancer have developed as an alternative approach to disease subtyping and risk stratification.

Since 2010, several studies have published genetic signatures in prostate cancer, describing both indolent [7] and aggressive disease [8], with generally very little overlap between them (Figure 1), although some have included common features $[8,9]$. Recently, we identified five distinct groups within prostate cancer, each with its own molecular fingerprint [10]. The two patient groups with the most dramatic genetic changes had the worst prognosis; indeed the genetic signatures for these groups

\section{KEYWORDS}

- clinical medicine $\bullet$ disease stratification genomics $\bullet$ gene signature $\bullet$ prostate cancer - translational research

“...[genetic] approaches are likely to form the basis of 'next-generation' screening..." 


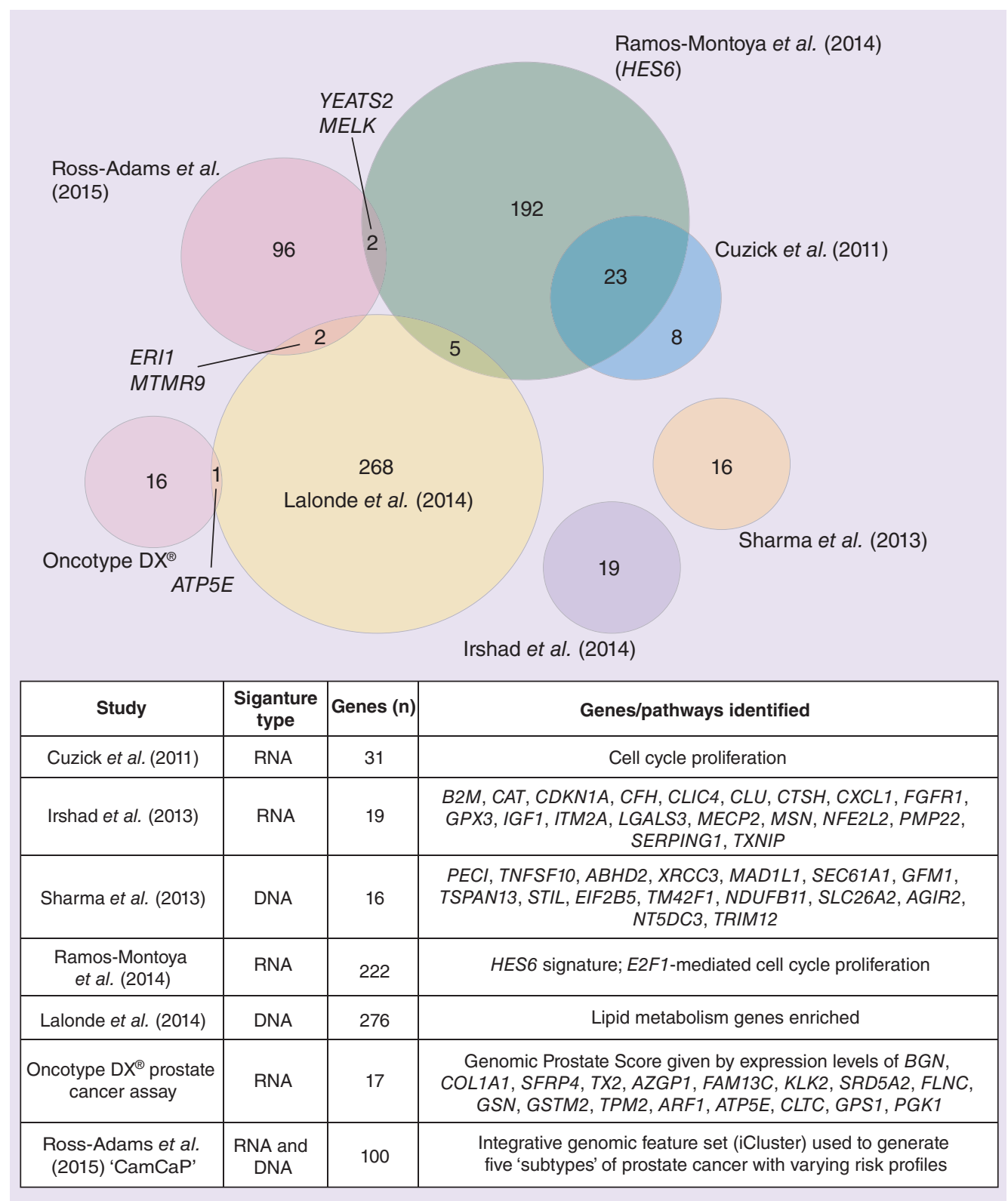

Figure 1. Published prostate cancer gene signatures and the extent of overlap.

Reproduced with permission from [10].

were better predictors of outcome than either PSA, Gleason score or tumor stage. Despite this exciting advance, we know that a one-sizefits-all approach to treating prostate cancer does not work, and it is similarly unlikely that one signature will answer all genetic risk questions. Furthermore, these approaches need to be applied to biopsy material. So far, molecular profiling studies have largely used tissue from radical prostatectomy operations. This has the advantage of yielding high concentrations of good quality material, but is biased by the fact that the decision to treat with major surgery has already been made in this patient group. An alternative could be to work with tissue from the earliest point of sampling - the diagnostic biopsy. This has proved more challenging due to the limited material provided by a biopsy, and clonal heterogeneity [11-13] means it is difficult to be sure whether the focus of cancer sampled is the dominant lesion in the prostate. Indeed, this latter problem may also confound sampling from prostatectomy specimens, depending on the approach used and may in part account for 
the scant overlap between the several prostate cancer gene signatures identified so far.

The use of circulating tumor DNA (ctDNA) as a marker to both predict and monitor cancer progression, or response to treatment, is an exciting area of research. Disease stratification studies may provide a starting point for identifying genetic markers amenable to this approach. Cell-free screening methods are also more appealing to patients, with liquid biopsies being far less invasive, risky or painful and with fewer side effects than needle biopsies. Further, there is the additional benefit of greatly increased sensitivity to detect tumor heterogeneity, since mutations from every tumor focus should be detected, in contrast to tissue biopsy. ctDNA studies so far have generally used a 'candidate gene' approach, since it is still technically challenging and prohibitively expensive to sequence a significant proportion of the genome (DNA) or transcriptome (RNA) at the depth required to be able to reliably identify rare variants. Despite this, several small studies have shown early success in ovarian, breast [14], and bowel among other cancers [15]. Most recently, the first study to predict resistance to abiraterone in patients prostate cancer was reported, following targeted sequencing of the androgen receptor gene from ctDNA in plasma from patients with advanced hormone-resistant prostate cancer [16]. Men with two specific mutations in their androgen receptor gene (T878A or L702H) demonstrated significant resistance to treatment, which suggests that early genetic testing of men could predict nonresponders.

Formalin-fixed paraffin-embedded (FFPE) tissues are still an under-used resource in genomic studies generally, despite almost every clinical research group typically having access to hundreds of such samples. Because of their age, they often have the advantage of far longer clinical follow-up periods than fresh-frozen tissues. This is invaluable in evaluating the long term prognostic utility of gene signatures and is particularly relevant to prostate cancer, which is generally diagnosed later in life and typically slow growing. Several companies now offer off-the-shelf DNA and RNA 'next-generation sequencing' kits that are specifically optimized for FFPE-derived samples and relatively easy to implement in a typical molecular biology laboratory. We will, no doubt, soon see cohorts emerge with much longer follow-up from research groups with access to next-generation sequencing facilities, and look forward to the molecular insights they offer.

There has been growing demand for the findings of genome-wide association studies in common, complex disorders to progress from risk-associated variants to clinically useful applications [17]. We can expect the efforts of prostate cancer researchers to develop in a similar vein, from merely identifying molecular signatures as 'labels' of good or bad outcome patient groups, to investigating the functional consequences or roles of specific genes or epigenetic features (e.g., methylation) in disease development, progression or treatment to resistance.

Relatively few molecular profiling studies have been undertaken in black African men, a group with typically high rates of prostate cancer, and more aggressive forms of the disease, even when other socio-economic factors are considered [18]. Asian men, by contrast, have much lower rates of prostate cancer. In order for us as a research and medical community to gain a better understanding of the molecular mechanisms underlying like prostate cancer (and other common, complex diseases), these ethnic groups should surely be given the same attention as their white North American or European counterparts. Genomic data generated from such diverse cohorts would prove highly informative, and should ideally be incorporated into existing bioinformatic portals such as CBioPortal [19] alongside International Cancer Genetics Consortium (ICGC) [20] and The Cancer Genome Atlas (TCGA) [21] data.

Technologies have developed rapidly since the early days of linkage disequilibrium studies to identify disease risk variants, and many junior researchers now take for granted that RNA-, DNA- and ChIP-seq data will be made publicly available in easily accessible repositories like GEO, TCGA, ENCODE, exome aggregation consortium (ExAC) [22] and many others. These technologies have vastly increased the data available to us; the effects are twofold:

- First, an explosion of information that requires ever-increasing reliance on specialized advanced bioinformatic analyses to meaningfully interpret the data. Indeed, entire careers can be forged in the synthesis and analysis of terabytes of these biological data. This trend is likely to continue, and it would be gratifying to wet-lab biologists and clinicians alike if a proportion of large funding awards were tagged for the specific training of bioinformaticians to "...we know that a one-size-fits-all approach to treating prostate cancer does not work [in prostate cancer]... 
face this challenge, with proper recognition of the vital contribution they make;

- Second, studies now need such large numbers of patient samples to detect increasingly rare variants that multicenter (and multidisciplinary) approaches are becoming standard. This risks excluding researchers located in areas with less easy access to resources and/or funding, who may nonetheless have relevant, interesting and genomically informative cohorts for study. We risk over-homogenization if we only investigate cohorts that are easy to recruit. Greater collaboration between more diverse research groups and disciplines are therefore likely to benefit the community as a whole, and could accelerate translational research efforts.

Ultimately, molecular profiling in prostate cancer is a means to an end (even though disease stratification can be an interesting and technically exciting end in itself). Historically, developments in prostate cancer research have lagged behind work in breast cancer, where clinically useful biomarkers derived from molecular profiling studies/stratification projects have translated into druggable targets that benefit specific patient groups. For example, trastuzumab (Herceptin ${ }^{\circledR}$ ) is used to treat HER2-positive breast cancer patients [23], while tamoxifen is used to treat ER-positive breast cancers [24]. Recently, a small Phase II clinical trial in 50 men with metastatic hormone-resistant prostate cancer reported that patients with defects in specific DNA-repair genes showed much greater response to olaparib (a PARP inhibitor) than men without such gene defects [25]. We can anticipate more work in this area for prostate cancer, and research collaborations between academia, pharma and biotech present an opportunity to combine the relative research freedoms of academia - where potential drug targets can be identified - with the expertise of industrial partners, who have the infrastructure and financial resources needed to develop promising targets and bring a drug to market.

It is now time to deliver on the promises of stratified medicine in prostate cancer: we need to better predict the likely course of disease in individual men; we need to identify those men who will respond to specific treatments; and we need to identify nonresponders early and understand likely mechanisms of such nonresponse in relevant individuals. Collectively, we need to build on our existing datasets to identify biomarkers that deliver added value to existing clinical tools and validate these in cohorts that include patients from as many ethnicities as possible. Emerging methods for noninvasive analysis of circulating tumor DNAs, and improved technologies for analyzing historic FFPE samples present a way of making rapid progress, bringing personalized medicine closer to reality in prostate cancer.

\section{Disclaimer}

The content is entirely our own, and does not necessarily represent the official views of Cardiff University or Addenbrooke's Hospital.

\section{Financial \& competing interests disclosure}

The authors have no relevant affiliations or financial involvement with any organization or entity with a financial interest in or financial conflict with the subject matter or materials discussed in the manuscript. This includes employment, consultancies, honoraria, stock ownership or options, expert testimony, grants or patents received or pending, or royalties.

No writing assistance was utilized in the production of this manuscript.

\section{References}

1 Schroder FH, Hugosson J, Roobol MJ et al. Screening and prostate-cancer mortality in a randomized European study. N. Engl. J. Med. 360(13), 1320-1328 (2009).

2 Andriole GL, Crawford ED, Grubb RL et al. Mortality results from a randomized prostate-cancer screening trial. N. Engl. J. Med. 360(13), 1310-1319 (2009).

3 Lane JA, Donovan JL, Davis M et al. Active monitoring, radical prostatectomy, or radiotherapy for localised prostate cancer: study design and diagnostic and baseline results of the ProtecT randomised Phase 3 trial. Lancet Oncol. 15(10), 1109-1118 (2014).

4 D'amico AV, Whittington R, Malkowicz SB et al. Biochemical outcome after radical prostatectomy, external beam radiation therapy, or interstitial radiation therapy for clinically localized prostate cancer. JAMA 280(11), 969-974 (1998).

5 Gronberg H, Adolfsson J, Aly M et al. Prostate cancer screening in men aged 50-69 years (STHLM3): a prospective population-based diagnostic study. Lancet Oncol. 16(16), 1667-1676 (2015).
6 Lamb AD, Bratt O. Towards "nextgeneration" prostate cancer screening. Lancet Oncol. 16(16), 1579-1580 (2015).

7 Irshad S, Bansal M, Castillo-Martin M et al. A molecular signature predictive of indolent prostate cancer. Sci. Transl. Med. 5(202), 202ra122 (2013).

8 Ramos-Montoya A, Lamb AD, Russell R et al. HES6 drives a critical AR transcriptional programme to induce castration-resistant prostate cancer through activation of an E2F1-mediated cell cycle network. EMBO Mol. Med. 6(5), 651-661 (2014). 
J, Swanson GP, Fisher G et al. Prognostic value of an RNA expression signature derived from cell cycle proliferation genes in patients with prostate cancer: a retrospective study. Lancet Oncol. 12(3), 245-255 (2011)

10 Ross-Adams H, Lamb AD, Dunning MJ et al. Integration of copy number and transcriptomics provides risk stratification in prostate cancer: a discovery and validation cohort study. EBioMedicine 2(9), 1133-1144 (2015).

11 Cooper CS, Eeles R, Wedge DC et al. Analysis of the genetic phylogeny of multifocal prostate cancer identifies multiple independent clonal expansions in neoplastic and morphologically normal prostate tissue. Nat. Genet. 47(4), 367-372 (2015).

12 Gundem G, Van Loo P, Kremeyer B et al. The evolutionary history of lethal metastatic prostate cancer. Nature 520(7547), 353-357 (2015).

13 Hong MK, Macintyre G, Wedge DC et al. Tracking the origins and drivers of subclonal metastatic expansion in prostate cancer. Nat. Commun. 6, 6605 (2015).

14 Forshew T, Murtaza M, Parkinson C et al. Noninvasive identification and monitoring of cancer mutations by targeted deep sequencing of plasma DNA. Sci. Transl. Med. 4(136), 136ra168 (2012).

15 Bettegowda C, Sausen M, Leary RJ et al. Detection of circulating tumor DNA in early- and late-stage human malignancies. Sci. Transl. Med. 6(224), $224 \mathrm{ra} 224$ (2014).

16 Romanel A, Tandefelt DG, Conteduca V et al. Plasma AR and abiraterone-resistant prostate cancer. Sci. Transl. Med. 7(312), 312re310 (2015).

17 Freedman ML, Monteiro AN, Gayther SA et al. Principles for the post-GWAS functional characterization of cancer risk loci. Nat. Genet. 43(6), 513-518 (2011).

18 Lloyd T, Hounsome L, Mehay A, Mee S, Verne J, Cooper A. Lifetime risk of being diagnosed with, or dying from, prostate cancer by major ethnic group in England 2008-2010. BMC Med. 13171 (2015).
19 cBioPortal. www.cbioportal.org

20 International Cancer Genome Consortium. https://icgc.org

21 The Cancer Genome Atlas. http://cancergenome.nih.gov

22 Broad Institute. www.exac.broadinstitute.org

23 Piccart-Gebhart MJ, Procter M, LeylandJones B et al. Trastuzumab after adjuvant chemotherapy in HER2-positive breast cancer. N. Engl. J. Med. 353(16), 1659-1672 (2005).

24 The Breast International Group (BIG) 1-98 Collaborative Group, Thurlimann B, Keshaviah A et al. A comparison of letrozole and tamoxifen in postmenopausal women with early breast cancer. N. Engl. J. Med. 353(26), 2747-2757 (2005).

25 Mateo J, Carreira S, Sandhu S et al. DNA-repair defects and olaparib in metastatic prostate cancer. N. Engl. J. Med. 373(18), 1697-1708 (2015). 